\title{
Comparative study on identification of healthy and osteoarthritic articular cartilages by fourier transform infrared imaging and chemometrics methods
}

\author{
Zhi-Hua Mao**, Yue-Chao Wu**, Xue-Xi Zhang*, Hao Gao* \\ and Jian-Hua Yin*, ${ }^{*}$ \\ *Department of Biomedical Engineering \\ Nanjing University of Aeronautics and Astronautics \\ 29 Yudao St, Nanjing, 210016 Jiangsu, P.R. China \\ †yin@nuaa.edu.cn
}

Received 13 April 2016

Accepted 19 September 2016

Published 27 October 2016

\begin{abstract}
Two discriminant methods, partial least squares-discriminant analysis (PLS-DA) and Fisher's discriminant analysis (FDA), were combined with Fourier transform infrared imaging (FTIRI) to differentiate healthy and osteoarthritic articular cartilage in a canine model. Osteoarthritic cartilage had been developed for up to two years after the anterior cruciate ligament (ACL) transection in one knee. Cartilage specimens were sectioned into $10 \mu \mathrm{m}$ thickness for FTIRI. A PLS-DA model was developed after spectral pre-processing. All IR spectra extracted from FTIR images were calculated by PLS-DA with the discriminant accuracy of $90 \%$. Prior to FDA, principal component analysis (PCA) was performed to decompose the IR spectral matrix into informative principal component matrices. Based on the different discriminant mechanism, the discriminant accuracy $(96 \%)$ of PCA-FDA with high convenience was higher than that of PLSDA. No healthy cartilage sample was mis-assigned by these two methods. The above mentioned suggested that both integrated technologies of FTIRI-PLS-DA and, especially, FTIRI-PCA-FDA could become a promising tool for the discrimination of healthy and osteoarthritic cartilage specimen as well as the diagnosis of cartilage lesion at microscopic level. The results of the study would be helpful for better understanding the pathology of osteoarthritics.
\end{abstract}

Keywords: Articular cartilage; osteoarthritis; Fourier transform infrared imaging; partial least squares discriminant analysis; Fisher's discriminant analysis.

$\dagger$ Corresponding author.

\$These two authors contributed equally to this study.

This is an Open Access article published by World Scientific Publishing Company. It is distributed under the terms of the Creative Commons Attribution 4.0 (CC-BY) License. Further distribution of this work is permitted, provided the original work is properly cited. 


\section{Introduction}

Articular cartilage is a thin layer of dense connective tissue, which covers the subchondral bone in a diarthrodial joint, to provide the joint with the functions of load-bearing, low friction and wearing resistance. ${ }^{1,2}$ Cartilage can be structurally sub-divided into three successive zones across its thin thickness: superficial zone (SZ), transitional zone (TZ) and radial zone (RZ), ${ }^{3,4}$ each having clear differences in structural and molecular properties.

Osteoarthritis (OA) is a chronic disease that is noted for the gradual erosion of articular cartilage and the progressive disability of joint. ${ }^{5}$ Although it is a common disease, a sensitive and accurate diagnosis procedure for the early OA is still unavailable in the clinics.

As a powerful analytical tool, Fourier transform infrared imaging (FTIRI) has been used in the laboratories to detect the molecular and structural changes in biological tissues with high sensitivity and resolution. When FTIRI was applied to articular cartilage, the characteristic absorption bands such as amide I (1700-1600 $\left.\mathrm{cm}^{-1}\right)$, amide II (1600$\left.1500 \mathrm{~cm}^{-1}\right)$, amide III $\left(1300-1200 \mathrm{~cm}^{-1}\right)$, and sugar $\left(1125-1000 \mathrm{~cm}^{-1}\right)$ could be used to qualitatively determine the content and anisotropic changes of collagen and proteoglycan (PG). ${ }^{6,7}$ Because of the substantial overlapping among these IR characteristic bands, conventional FTIRI has difficulties to obtain the quantitative information in cartilage tissue. The detection ability of FTIRI can be improved by the use of several chemometric approaches ${ }^{6,8,9}$ including principal component analysis (PCA), which is the most general technique of dimensional reduction by searching for the best linear combination among the variables.

Partial least squares-discriminant analysis (PLS-DA) is a classification method in spectral analysis, ${ }^{10,11}$ which is based on the PLS algorithm. The procedure makes use of the entire spectra rather than the individual absorption bands. The complex relationships between spectral matrix (prediction matrix) and categorical matrix could be mapped out by PLS-DA, which has powerful discriminant ability since it extracts information from both spectral matrix and categorical matrix. ${ }^{12,13}$

Fisher's discriminant analysis (FDA) is an analytical technique for classification, which is a linear discriminant analysis based on the variance analysis so that several groups or classes could be discriminated as clearly as possible. ${ }^{14-16}$ In PCA-FDA, PCA was first employed to decompose the spectral matrix into the score matrix and loading matrix. Then, the classification function of FDA would be built on the basis of the score matrix.

Following our previous work on the concentration prediction of collagen and PG in healthy and OA cartilage by FTIRI-PLS, ${ }^{17}$ two diagnosing methods of PLS-DA and PCA-FDA were employed to differentiate the healthy and $\mathrm{OA}$ cartilage. The classification results and mechanism would be compared and discussed for accurately understanding the discrimination ability of both methods.

\section{Materials and Methods}

\subsection{Sample preparation}

The articular cartilage specimens were harvested from 10 mature dogs (five healthy and five OA) after they were euthanized by injecting an intravenous overdose of sodium pentobarbital. The five OA canines underwent an anterior cruciate ligament (ACL) transection surgery in one of the knee joints up to two years ago, which resulted in a classical arthritis. ${ }^{18-20}$ The protocols and handling of the animals had been approved by the institutional animal care committees. ${ }^{21}$

Upon harvesting, the medial tibias in the knee joints were cut by a table saw with a diamond blade. The cartilage specimens contained the fullthickness cartilage that was still attaching to the subchondral bones, from the tibial locations that were covered with meniscus. The size of the cartilage-bone blocks was about $2 \mathrm{~mm} \times 2 \mathrm{~mm} \times 2 \mathrm{~mm}$. After washed in saline and quickly frozen by liquid nitrogen, these blocks were sectioned into $10 \mu \mathrm{m}$ thick sections with a cryostat (Leica CM 1950) at $-20^{\circ} \mathrm{C}$, in a vertical cutting direction towards the cartilage surface. The thin sections were placed on the MirrIR slides (Kevley Technologies, Chesterland, $\mathrm{OH}$ ) and dried in the air before FTIRI experiments.

\subsection{FTIR imaging}

FTIRI experiments were carried out on PerkinElmer Spotlight-300 infrared imaging system, which includes a FTIR spectrometer (Spectrum One) and an infrared microscope. The MirrIR slides with cartilage sections were placed on the scanning stage 
of the FTIRI system. Infrared imaging data was collected by a mercury cadmium telluride (MCT) array detector at $6.25 \mu \mathrm{m} /$ pixel size and $8 \mathrm{~cm}^{-1}$ wavelength spacing (namely $16 \mathrm{~cm}^{-1}$ spectral resolution) over a mid-infrared range of $4000-744 \mathrm{~cm}^{-1}$. The background data of the MirrIR slide was also collected in the same spectral range for the purpose of background correction. Two scans per pixel were performed for the background and the cartilage sections, respectively.

IR spectra were extracted from the FTIR images of all 10 articular cartilage samples. Each spectrum was averaged from the specifically selected areas in FTIR images, from the areas of $100 \mu \mathrm{m}$ depth beneath the cartilage surface with $10 \mu \mathrm{m}$ intervals. 10 spectra were extracted from each sample. The final raw data was 100 spectra, which were sequentially numbered from 1 to 100 .

\subsection{PLS-DA method}

PLS-DA was carried out in Unscrambler $X$ software (CAMO Software Inc, Woodbridge, NJ). A PLSDA model was built to correlate the IR spectral matrix to the categorical matrix that was actually a category vector designating the classes of the cartilage with 0 and $1 .{ }^{10,12}$ Specifically, 1 in the categorical matrix indicates that its corresponding spectrum belongs to the healthy group. In contrast, 0 indicates that the spectra come from the OA group. In addition, a cut-off value was established and set as 0.5 in the PLS-DA model. If the prediction value is larger than 0.5 , the sample belongs to Class 1 (healthy group), otherwise it is assigned to Class 0 (OA group).

To decrease the heterogeneity of biological tissues, as well as to ensure the universal statistical significance, 18 spectra were randomly selected from the 100 spectra to constitute the training dataset for the PLS-DA model that includes at least one spectrum from each sample. The remaining spectra (82 spectra), serving as the prediction matrix, were prepared for the prediction.

Spectral pre-processing, including multiplicative scatter correction (MSC), normalization and baseline offset, were performed to improve the reliability and stability of PLS-DA model. Leave-one-out cross validation was carried out to evaluate the mapping relationship between spectral matrix and categorical matrix of the training dataset. ${ }^{22}$ The fitting coefficient (Pearson's $r$ ) of the actual categorical values and PLS-predicted categorical values for the training dataset is 0.946 . The calculated results indicated an excellent fitting relationship $(r=0.946)$ between the spectral matrix and categorical matrix, and a well representation of this PLS-DA model.

\subsection{PCA-FDA method}

PCA-FDA was performed in SPSS Statistics 20 software (IBM Software, USA). PCA was carried out without pre-processing of the IR spectral dataset; PC factor was determined by the cumulative percent variance contribution. The FDA principles are to maximize covariance in one class and minimize covariance among different classes. ${ }^{15,16,23}$ Classification function of FDA was built based on PCA result through covariance analysis that was shown below.

If the PC factor was set as $2, \boldsymbol{Y}_{1}$ and $\boldsymbol{Y}_{2}$ are the calculated score values by the classification functions of (1) and (2) for each spectrum, $\boldsymbol{X}_{1}$ and $\boldsymbol{X}_{2}$ are the score values of $\mathrm{PC} 1$ and $\mathrm{PC} 2$.

$$
\begin{gathered}
\boldsymbol{Y}_{1}=0.735 \boldsymbol{X}_{1}+2.101 \boldsymbol{X}_{2}-1.582 \\
\boldsymbol{Y}_{2}=-0.735 \boldsymbol{X}_{1}-2.101 \boldsymbol{X}_{2}-1.582
\end{gathered}
$$

All 100 spectra were calculated by the PCA-FDA classification function and then sequenced from 1 to 100 with the same order in PLS-DA. The cut-off threshold is 0 , which was determined by the classification function of PCA-FDA. If the score of the spectrum is less than 0, it belongs to healthy group; otherwise it belongs to OA group.

\subsection{Statistical analysis}

In order to identify the individual sample from healthy and OA group, statistical analysis was performed for the PLS-DA categorical values and the values of the classification function in PCA-FDA, respectively, by using the software of Origin 8.0 (OriginLab Coop, MA). The data of control sample for healthy and OA cartilages were compared with those of other healthy and OA samples using One-Way ANOVA, respectively. If the $p$-value is below 0.05 , it means that there was a significant difference between the two compared samples.

\section{Results}

Figure 1 showed a spectrum extracted from the FTIR image of one cartilage specimen over a range 


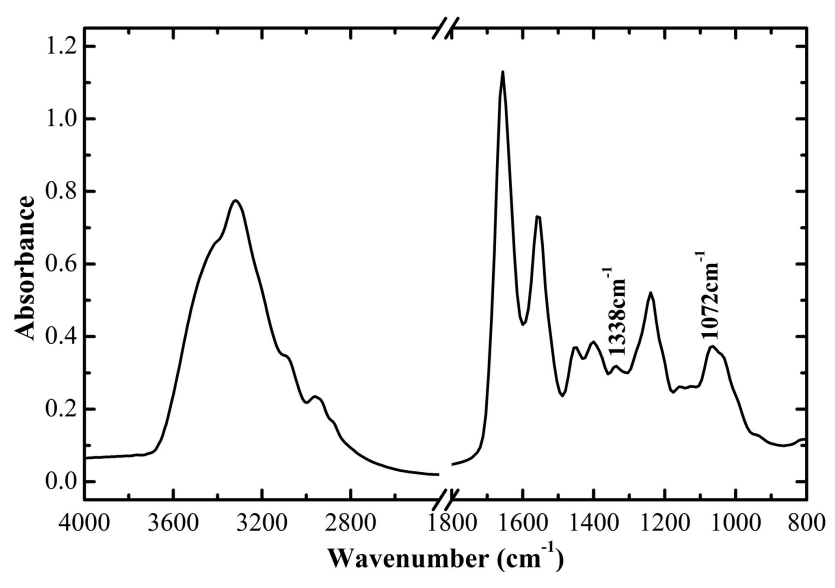

Fig. 1. IR spectrum extracted from FTIR image of a cartilage section.

of $4000-800 \mathrm{~cm}^{-1}$. The characteristic absorption bands marked in the figure were assigned to collagen $\left(1338 \mathrm{~cm}^{-1}\right)$ and PG $\left(1072 \mathrm{~cm}^{-1}\right)$, respectively. The characteristic absorption images of the two peaks could be used to qualitatively represent the distributions of collagen and PG. ${ }^{6}$

Figure 2 displayed the images of a healthy cartilage section and an OA cartilage section, including the total absorption images characteristic absorption images of PG $\left(1072 \mathrm{~cm}^{-1}\right)$ and visible images. The dark circles with irregular shape in the visible

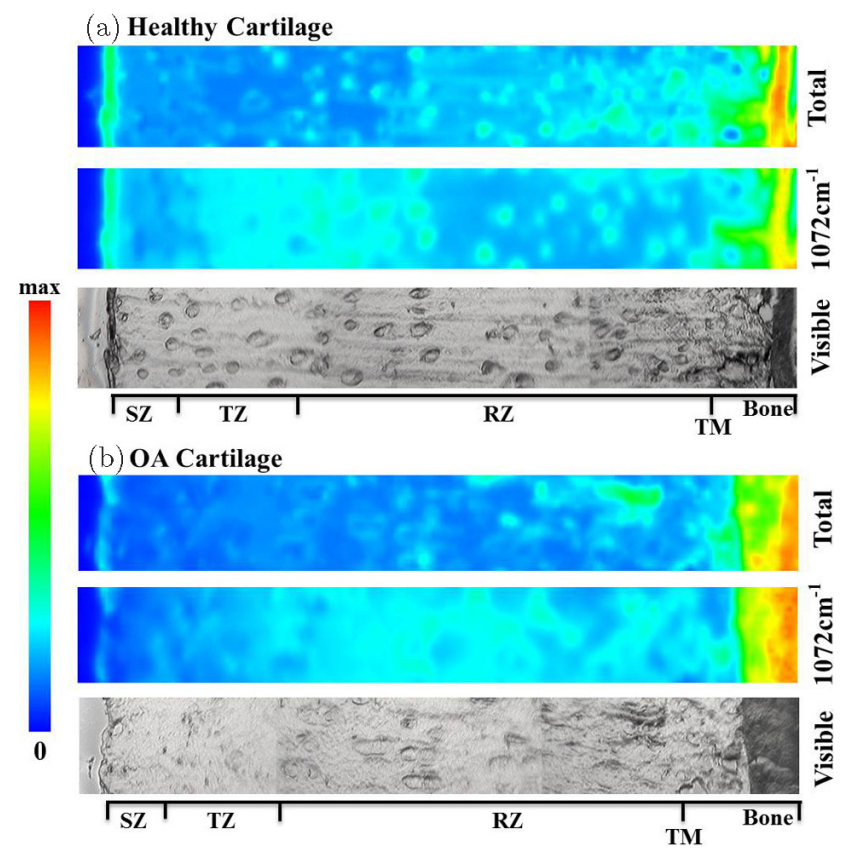

Fig. 2. FTIR images of total absorption images $1072 \mathrm{~cm}^{-1}$ images and visible images of a healthy (a) and an OA (b) cartilage, respectively. The absorption max for total absorption images and $1072 \mathrm{~cm}^{-1}$ images were 1.5 and 2.0 , respectively. images were the chondrocytes, which were less visible in SZ and TZ of the OA cartilage, as compared to the healthy cartilage. In addition, the absorption intensity in SZ and TZ of OA cartilage image was lower than that of the healthy cartilage, suggesting a clear PG loss in these regions. ${ }^{6,24}$ The IR spectra were extracted from SZ and TZ (100 $\mu \mathrm{m}$ depth beneath the cartilage surface) for the identification analysis.

In PLS-DA and PCA, the cumulative percent variance contributions of $\mathrm{PC}$ scores from $\mathrm{PC} 1$ to PC7 were shown in Fig. 3(a). The contribution of $\mathrm{PC} 1$ and $\mathrm{PC} 2$ in PLS-DA and PCA was $88.93 \%$ and $91.76 \%$, respectively. In PCA, PC1 versus PC2 scores were plotted in Fig. 3(b) for all healthy $(\bullet)$ and OA $(\boldsymbol{\Delta})$ cartilage spectra (100 spectra). The

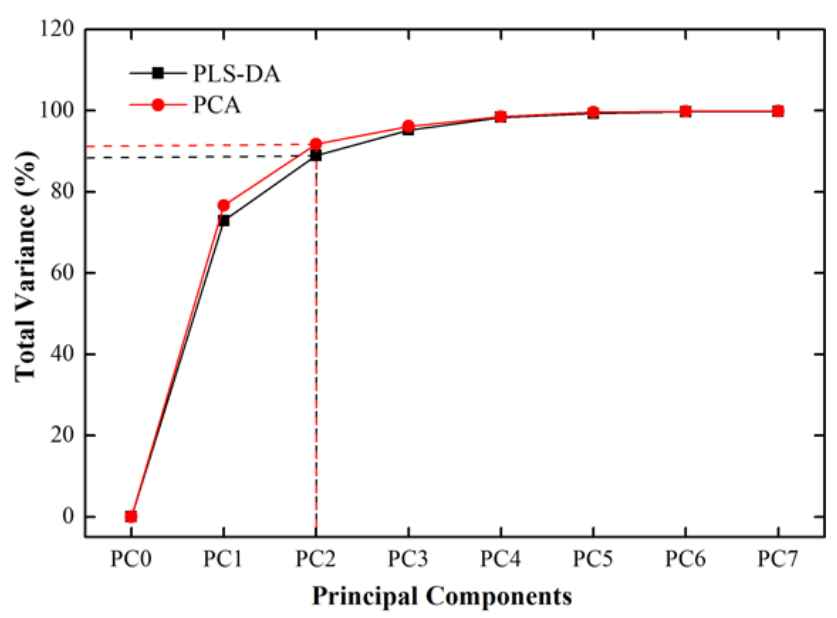

(a)

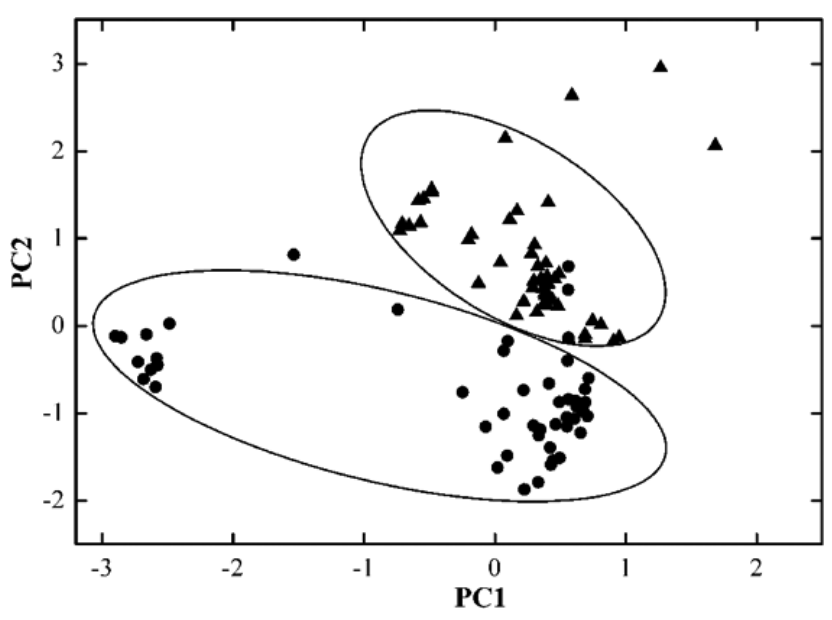

(b)

Fig. 3. (a) Cumulative percent variance contributions of principal components in the PLS-DA and PCA model. (b) PCA scores plot (PC1 versus PC2) for the analysis of the FTIR spectra originated from $(\bullet)$ healthy cartilage and $(\boldsymbol{\Lambda})$ OA cartilage. 


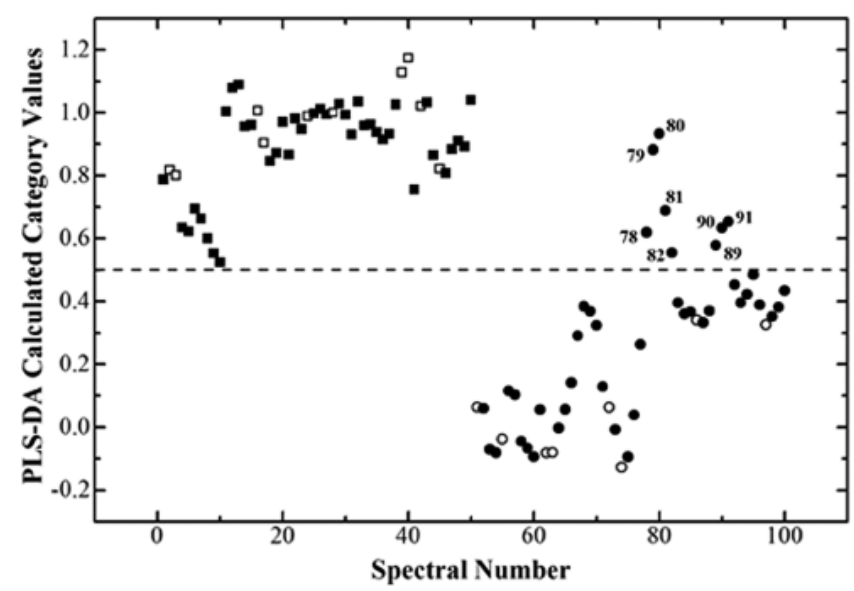

(a)

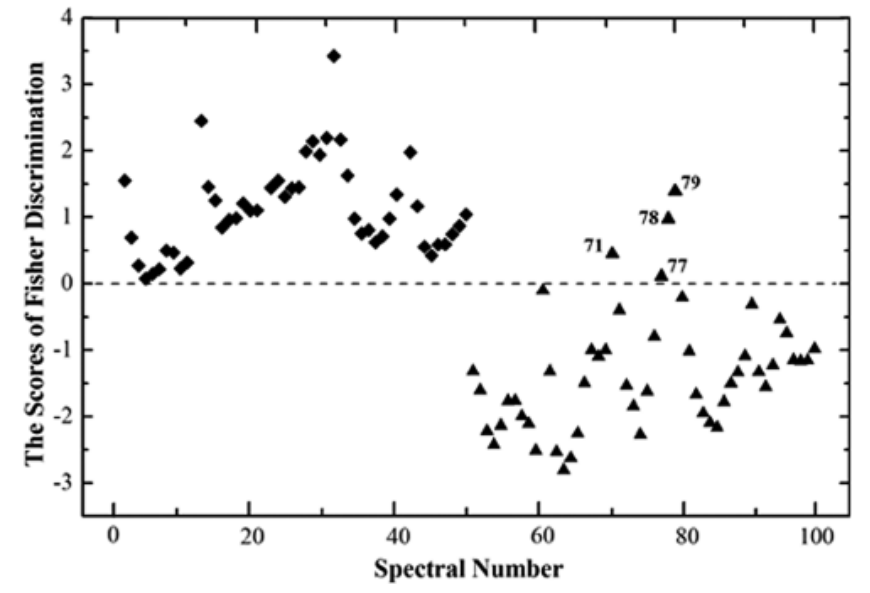

(b)

Fig. 4. (a) PLS-DA categorical values of training dataset and prediction matrix that were numbered from 1 to 100 . Healthy ( $\square$ ) and OA (०) cartilage spectra of training dataset were predicted for cross validation, healthy $(\boldsymbol{\square})$ and OA (•) cartilage spectra of prediction matrix were predicted by PLS-DA. (b) Scores calculated by PCA-FDA method. Healthy $(\downarrow)$ and OA (ム) cartilage spectra were sequenced with the same order in PLS-DA. Mis-assigned spectra in PLS-DA and PCA-FDA were marked with their sequence numbers and drawn above the dash lines.

healthy and OA spectra can generally be distinguished into two clusters. Two ellipses that added in Fig. 3(b) were drawn as the clustering center of PC scores with $95 \%$ confidence intervals.

Figure 4 displayed the PLS-DA categorical values of the 100 spectra (Fig. 4(a)) and the values of the classification function in PCA-FDA (Fig. 4(b)). Spectra \#1-50 were originated from the healthy group, which were drawn as the solid and hollow square points in Fig. 4(a) (PLS-DA method)

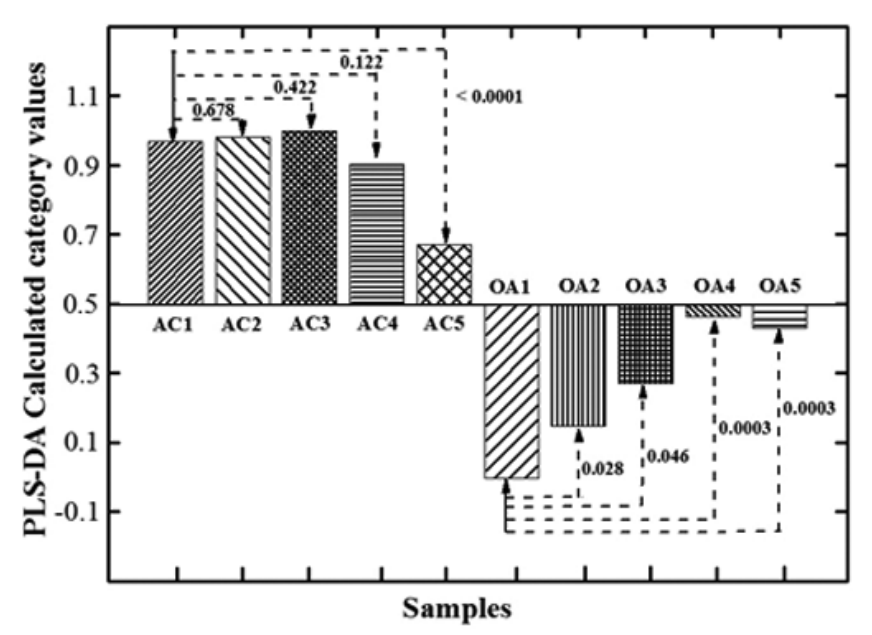

(a) and rhombus points in Fig. 4(b) (PCA-FDA method), respectively; Spectra 51-100 came from the OA cartilage, shown as the round dots in Fig. 4(a) and triangles in Fig. 4(b), respectively.

For PLS-DA method, the prediction accuracies of training dataset and prediction matrix were $100 \%$ and $90 \%$ (correctly assigned spectra in prediction matrix/all spectra in prediction matrix $=74 / 82$ ), respectively. All healthy cartilage spectra were correctly distinguished by the PLS-DA both in

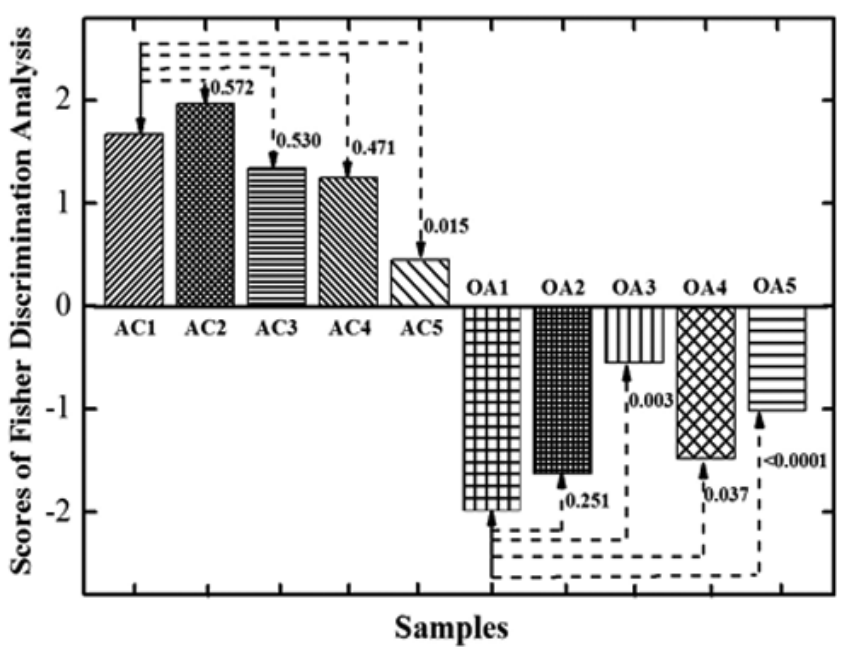

(b)

Fig. 5. Statistical analysis of PLS-DA categorical values (a) and the score values of PCA-FDA (b) for healthy and OA groups, respectively. The healthy cartilages were sequenced from AC1 to AC5, as well as the OA cartilages from OA1 to OA5. AC1 and OA1 were regarded as control in the corresponding group for statistical analysis, respectively. Each of the arrow pair indicates the statistical analysis between the control and comparative samples where the corresponding $p$-value is given around the lines. 
training dataset or prediction matrix; however eight spectra from the OA cartilages were mis-assigned by PLS-DA (OA3, OA4, and OA5 in Fig. 5). The predicted categorical values of the eight misassigned spectra above the cut-off value of 0.5 were drawn as the solid circles above the dash line and marked with their sequence numbers.

The values in Fig. 4(b) were calculated by the classification function in PCA-FDA. They showed the similar distribution tends when compared to Fig. 4(a). However, discrimination accuracy obtained by the PCA-FDA was higher than that by PLS-DA. The discrimination accuracy of PCA-FDA was $96 \%$ and $100 \%$ for all 100 spectra and healthy group, respectively. Four OA spectra were misassigned by PCA-FDA, which originated from OA3.

One-way ANOVA was performed for the PLSDA and PCA-FDA results, Statistical analysis based on the PLS-DA categorical values and the score values of PCA-FDA was shown in Fig. 5. It displays the similar tendency for the healthy group, that is, the differences among the healthy cartilages were unobvious in the whole, except of AC5. All healthy cartilage used for the analysis had similar character and quality. However, statistical analysis for the OA group showed opposite aspect, with obvious difference existing among the five OA cartilages, which suggests that individual difference easily happens among the OA samples although all of the OA cartilage were harvested from the 2-year ACL-transection knee joints.

\section{Discussion}

Two discrimination algorithms were combined with FTIRI data analysis to successfully identify the healthy and OA cartilages with high accuracy. PLSDA and PCA-FDA in this project were sufficiently sensitive to small changes in principal macromolecule content between the healthy and OA cartilage samples. No healthy cartilage was mis-assigned in both PLS-DA and PCA-FDA. According to the sequence number of the 100 spectra, the misassigned cases originated from the regions of 0-20 $\mu \mathrm{m}$ and $80-100 \mu \mathrm{m}$ for the OA cartilages.

\subsection{Reasons for the mis-assignments}

In general, it is known that even for a well-controlled group of animals, the development of the tissue damages might not be uniform. The OA damage of an individual OA sample with the misassigned spectra could be weaker than that of other OA samples, so that the cartilage degeneration did not develop into the region $(80-100 \mu \mathrm{m})$ for these mis-assigned sections. PG loss could be unobvious in this area. ${ }^{6,24}$ On the other hand, individual OA sample could also be seriously damaged at SZ, so that it becomes difficult to define the actual surface of OA cartilage section. It may cause the last 2 or 3 spectra were actually extracted from much deeper location than $80-100 \mu \mathrm{m}$, where might be in RZ with less PG loss. ${ }^{6,25}$ These concentration variations could be the reasons for mis-assigning the spectra in the position of $80-100 \mu \mathrm{m}$.

In the case of the mis-assignment at the surface 0-20 $\mu \mathrm{m}$ region, the peripheral regions of cartilage sections may slightly curl or crimp during the airdrying of the histological process. Additionally, due to the changes of chondrocytes in OA cartilage, scattering effects might be stronger in SZ than those in the intermediate layer for individual OA sample. $^{26,27}$ These two reasons could lead to the misassignments in the $0-20 \mu \mathrm{m}$ position for OA cartilages. On the other hand, heterogeneity in the samples might also have smaller influence on the identification.

\subsection{PLS-DA and PCA-FDA}

PLS-DA and PCA-FDA employed different discrimination mechanisms to effectively identify the healthy and OA cartilages with high accuracies.

PLS-DA is developed from the PLS regression algorithm. The classification model was built based on the changes of the PG content between healthy and OA cartilage. ${ }^{6,28}$ The discrimination accuracy for the prediction matrix depended on the PLS regression model, the categorical matrix and the cut-off values (0.5) in the PLS-DA. Spectral preprocessing was applied in PLS-DA to reduce the negative effects (multiplicative scattering, diffuse reflection etc.) in the spectra.

PCA is a technique of dimensional reduction and FDA is a common linear classification algorithm. In PCA-FDA, 100 spectra were simplified by PCA into several PCs scores in the initial process. PC1 and PC2 scores were used for building the classification function in FDA method, which corresponded to the two principal components (collagen and PG) in the cartilage. No pre-processing was performed for the 100 spectra in PCA-FDA. The discrimination 
accuracy of PCA-FDA depended on the cut-off values ( 0$)$ and the values that were determined by the classification function.

The values calculated by PLS-DA and PCAFDA showed the similar distribution tends (Fig. 4). All mis-assigned cases originated from the OA cartilages in the regions of $0-20 \mu \mathrm{m}$ and $80-100 \mu \mathrm{m}$ and no healthy cartilage was mis-assigned. It was indicated that the principal component change could be identified by these two methods effectively. Both the classification models had high specificity, respectively. In PLS-DA, the value distribution of healthy cartilage was more stable, which means PLS-DA shows more advantage than PCA-FDA for healthy samples. On the contrary, the value of OA cartilage could be easily distinguished from healthy cartilage by PCA-FDA, especially for OA4 and OA5 in this case.

Specifically, PCA-FDA showed a better accuracy than PLS-DA when comparing the sequence numbers of mis-assigned spectra in PLS-DA and PCAFDA. All mis-assigned spectra (\#71, \#77, \#78, and \#79) in PCA-FDA originated from OA3 cartilage, while other OA cartilages were correctly identified by the PCA-FDA. However, several spectra that extracted from OA3 (\#78, \#79, \#80), OA4 (\#81, \#82, \#89, and \#90) and OA5 (\#91) were mis-assigned by the PLS-DA. The reasons mentioned below might cause the misjudgments. First, the cumulative percentage variance contribution in PCA-FDA was higher than PLS-DA, which means more information of the spectra has been remained in PCA-FDA. It was associated with the accuracy of the classification model directly. Second, PLS-DA model depends on the categorical matrix which represented the comprehensive change of two principal components rather than the content change of single component in OA cartilages. This suggested that the value calculated by PLS-DA might not perfectly reflect the fine change of single principal component in the specific areas mentioned above.

Leave-one-out cross validation was both carried out for the training matrix of PLS-DA and 100 spectra in PCA-FDA. The discrimination accuracy for PLS-DA and PCA-FDA in the cross validation method is $100 \%$ and $96 \%$. The results suggested that this study was obviously different from the previous work with normal PLS ${ }^{13,17,19,29}$ and sole PLS-DA. ${ }^{21}$

\section{Conclusion}

FTIRI-PLS-DA and FTIRI-PCA-FDA showed excellent diagnostic ability to distinguish between healthy and OA cartilage. PCA-FDA showed a higher discrimination accuracy $(96 \%)$ than that of PLS-DA (90\%). No healthy cartilage was misassigned by the two integrated methods. It is demonstrated that either FTIRI-PLS-DA or FTIRI-PCA-FDA would be a promising discrimination technique for differentiating healthy and $\mathrm{OA}$ cartilage. In future work, these methods could be extended to study a large number of samples to evaluate the discrimination ability among different grades of OA cartilages, to determine the clinical significance of FTIRI-PLS-DA and FTIRIPCA-FDA.

\section{Acknowledgment}

Jian-Hua Yin is grateful to (1) the National Natural Science Foundation of China for the grant of 61378087; (2) Natural Science Foundation of Jiangsu Province (BK20151478). Zhi-Hua Mao is grateful to the Open Funds for Graduate Innovation Lab of Nanjing University of Aeronautics and Astronautics (kfjj20150309) and Fundamental Research Funds for the Central Universities. The raw data acquisition in FTIRI was mostly carried out in the lab of Professor Yang Xia at Oakland University (Rochester, Michigan, USA). Professor Xia was supported by an NIH grant R01-AR052353 during the time of the data acquisition.

\section{References}

1. A. K. Williamson, A. C. Chen, R. L. Sah, "Compressive properties and function-composition relationships of developing bovine articular cartilage," J. Orthop. Res. 19, 1113-1121 (2001).

2. W. Wilson, J. M. Huyghe, C. C. van Donkelaar, "Depth-dependent Compressive Equilibrium Properties of Articular Cartilage Explained by its Composition," Biomech. Model. Mechanobiol. 6, 43-53 (2007).

3. N. Ramakrishnan, Y. Xia, A. Bidthanapally, M. Lu, "Determination of zonal boundaries in articular cartilage using infrared dichroism," Appl. Spectrosc. 61, 1404-1409 (2007).

4. J. I. Lee, Y. Xia, "Quantitative zonal differentiation of articular cartilage by microscopic magnetic 
resonance imaging, polarized light microscopy and Fourier-transform infrared imaging," Microsc. Res. Tech. 76, 625-632 (2013).

5. X. H. Bi, Y. Xia, M. P. Bostrom, N. P. Camacho, "Fourier transform infrared imaging spectroscopy investigations in the pathogenesis and repair of cartilage," Biochim. Biophys. Acta 1758, 934-941 (2006).

6. J. H. Yin, Y. Xia, M. Lu, "Concentration profiles of collagen and proteoglycan in articular cartilage by Fourier transform infrared imaging and principal component regression," Spectrochim. Acta A Mol. Biomol. Spectrosc. 88, 90-96 (2012).

7. Y. Xia, N. Ramakrishnan, A. Bidthanapally, "The depth-dependent anisotropy of articular cartilage by Fourier-transform infrared imaging (FTIRI)," Osteoarthr. Cartilage 15, 780-788 (2007).

8. L. Rieppo, J. Rieppo, J. S. Jurvelin, S. Saarakkala, "Fourier Transform Infrared Spectroscopic Imaging and Multivariate Regression for Prediction of Proteoglycan Content of Articular Cartilage," PLoS One 7, e32344 (2012).

9. Z. H. Mao, X. X. Zhang, Y. C. Wu, J. H. Yin, Y. Xia, "Fourier Transform Infrared Microscopic Imaging and Fisher Discriminant Analysis for Identification of Healthy and Degenerated Articular Cartilage," Chin. J. Anal. Chem. 43, 518-522 (2015).

10. A. D. Ghanate, S. Kothiwale, S. P. Singh, D. Bertrand, C. M. Krishna, "Comparative evaluation of spectroscopic models using different multivariate statistical tools in a multicancer scenario," $J$. Biomed. Opt. 16, 025003 (2011).

11. K. H. Wong, V. Razmovski-Naumovski, K. M. Li, G. Q. Li, K. Chan, "Differentiation of Pueraria lobata and Pueraria thomsonii using partial least square discriminant analysis (PLS-DA)," J. Pharm. Biomed. Anal. 84, 5-13 (2013).

12. R. G. Brereton, "Introduction to multivariate calibration in analytical chemistry," Analyst 125, 21252154 (2000).

13. M. P. O'Brien, M. Penmatsa, U. Palukuru, P. West, X. Yang, M. P. G. Bostrom, T. Freeman, N. Pleshko, "Monitoring the progression of spontaneous articular cartilage healing with infrared spectroscopy," Cartilage 6, 174-184 (2015).

14. H. C. Leo, L. R. Evan, D. B. Richard, "Fault diagnosis in chemical processes using Fisher discriminant analysis, discriminant partial least squares, and principal component analysis," Chemometr. Intell. Lab. 50, 243-252 (2000).

15. E. Ostrovsky, U. Zelig, I. Gusakova, S. Ariad, S. Mordechai, I. Nisky, J. Kapilushnik, "Detection of Cancer Using Advanced Computerized Analysis of Infrared Spectra of Peripheral Blood," IEEE Trans. Biomed. Eng. 60, 343-352 (2013).
16. D. Liu, X. J. Sun, C. Zhang, S. Y. Zhang, J. B. Zheng, R. Gurung, J. K. Du, J. S. Shi, Y. Z. Xu, Y. F. Zhang, J. G. Wu, " Evaluation of FTIR spectroscopy as diagnostic tool for colorectal cancer using spectral analysis," Spectrochim. Acta A Mol. Biomol. Spectrosc. 122, 288-294 (2014).

17. X. X. Zhang, Z. H. Mao, J. H. Yin, Y. Xia, "Determination of collagen and proteoglycan concentration in osteoarthritic and healthy articular cartilage by Fourier transform infrared imaging and partial least square," Vib. Spectrosc. 78, 49-53 (2015).

18. X. H. Bi, Y. Xia, M.P. G. Bostrom, D. Bartusik, S. Ramaswamy, K. W. Fishbein, R. G. Spencer, N. P. Camacho, "Fourier transform infrared imaging and MR microscopy studies detect compositional and structural changes in cartilage in a rabbit model of osteoarthritis," Anal. Bioanal. Chem. 387, 16011612 (2007).

19. S. L. Myers, K. D. Brandt, B. L. O'Connor, D. M. Visco, M. E. Albrecht. "Synovitis and osteoarthritic changes in canine articular cartilage after anterior cruciate ligament transection. Effect of surgical hemostasis," Arthritis Rheum. 33, 1406-1415 (1990).

20. J. H. Lee, F. Badar, BScE, J. Matyas, Y. Xia, "Detecting the Sub-tissue Zonal Changes in Glycosaminoglycan (GAG) Contents between Healthy and Early Osteoarthritic Tibial Cartilages by Nondestructive Imaging," http://www.ors.org/Transactions $/ 60 / 0373 . p d f$.

21. X. X. Zhang, J. H. Yin, Z. H. Mao, Y. Xia, "Discrimination of healthy and osteoarthritic articular cartilages by Fourier transform infrared imaging and partial least squares-discriminant analysis," J. Biomed. Opt. 20, 060501 (2015).

22. J. A. Westerhuis, H. C. J. Hoefsloot, S. Smit, D. J. Vis, A. K. Smilde, E. J. J. van Velzen, J.P. M. van Duijnhoven, F. A. van Dorsten, "Assessment of PLSDA cross validation," Metabolomics 4, 81-89 (2008).

23. P. Aberg, P. Geladi, I. Nicander, J. Hansson, U. Holmgren, S. Ollmar, "Non-invasive and microinvasive electrical impedance spectra of skin cancera comparison between two techniques," Skin. Res. Technol. 11, 281-286 (2005).

24. J. H. Yin, Y. Xia, "Proteoglycan concentrations in healthy and diseased articular cartilage by Fourier transform infrared imaging and principal component regression," Spectrochim. Acta A Mol. Biomol. Spectrosc. 133, 825-830 (2014).

25. E. David-Vaudey, A. Burghardt, K. Keshari, A. Brouchet, M. Ries, S. Majumdar, "Fourier Transform Infrared Imaging of focal lesions in human osteoarthritic cartilage," Eur. Cell. Mater. 10, 51-60 (2005). 
26. K. Yamamoto, T. Shishido, T. Masaoka, A. Imakiire, "Morphological studies on the ageing and osteoarthritis of the articular cartilage in C57 black mice," J. Orthop. Surg. 13, 8-18 (2005).

27. M. B. Goldring, "The role of the chondrocyte in osteoarthritis. Arthritis Rheum," Arthritis Rheum. 43, 1916-1926 (2000).

28. S. Saarakkala, P. Julkunen, P. Kiviranta, J. Mäkitalo, J. S. Jurvelin, R. K. Korhonen," Depth-wise progression of osteoarthritis in human articular cartilage: Investigation of composition, structure and biomechanics," Osteoarthr. Cartilage 18, 73-81 (2010).

29. A. Hanifi, X. Bi, X. Yang, B. Kavukcuoglu, P. C. Lin, E. DiCarlo, P. G. Spencer, M. P. G. Bostrom, N. Pleshko, "Infrared Fiber Optic Probe Evaluation of Degenerative Cartilage Correlates to Histological Grading," Am. J. Sports. Med.40, 2853-2861 (2012). 